Ulrich Dammann, Wissenschaftlicher Mitarbeiter der Forschungsstelle für Juristische Dokumentation, Frankfurt/M. D-6 Frankfurt, Senckenberganlage 31

Andrea Hasselkuß, Forschungsstelle für Juristische Informatik und Automation der Universität Bonn. D-53 Bonn-Beuel, Ringstraße 43

Claus-Jürgen Kaminski, Referendar, Gesellschaft für Mathematik und Datenverarbeitung mbH, Birlinghoven bei Bonn. D-53 Bonn-Beuel, Ringstraße 43

Hans Brinckmann, Dr. jur., Dipl.-Ing., Technische Hochschule Darmstadt. D-6079 Buchschlag, Hengstbachanlage 6

Hannes Rieser, Dr. phil., Universität Konstanz, Fachbereich Sprachwissenschaften. D-775 Konstanz, Jakob-Burckhardt-Straße

János S. Petöfi, Dr., Privatdozent, Universität Konstanz, Fachbereich Sprachwissenschaften. D-775 Konstanz, Jakob-Burckhardt-Straße

\title{
Beiträge in den nächsten Heften
}

Gerhard Wittkämper, Aspekte und Strukturen der Datenschutzgesetzgebung in der Welt; Gerhard Stadler, Die Anwendung der EDV und kybernetischer Methoden im Gesetzgebungsprozeß; Helga Stadler, Die Anwendung der EDV im Rechtsstudium und bei juristischen Prüfungen; Vladimir Vrecion, Anwendbarkeit der Informationstheorie im Recht; Dieter Goose, Operations Research und Verwaltungslehre; Diethard Zielinski, Ein Juristisches Informationssystem für die Bundesrepublik? Aviezri S. Fraenkel, The responsa full text retrieval project - phase II; Bernhard Vischer, Schutz der Privatsphäre und EDV; Bernhard von Linstow, Datenverarbeitung und Strafzumessung; Hélène Bauer-Bernet, Kommunikation zwischen Dokumentationssystemen: Theorie und praktische Erfahrungen; Leo Reisinger, Strukturtheorie des Rechts und EDV; Werner A. Schmidt, Grundsätzliche und methodische Probleme automativer Rechtsgestaltung bei einer Umstellung des Grundbuches auf elektronische Datenverarbeitung. 